\title{
Padrões ecológicos da comunidade de ácaros em euforbiáceas de um fragmento de mata Estacional Semidecidual, no Estado de São Paulo
}

\author{
Reinaldo José Fazzio Feres ${ }^{1.3}$, Renato Buosi ${ }^{2}$, Rodrigo Damasco Daud ${ }^{2}$ \& Peterson Rodrigo Demite ${ }^{2}$
}

Biota Neotropica $v 7$ (n2) - http://www.biotaneotropica.org.br/v7n2/pt/abstract?article +bn04907022007

\author{
Recebido em 23/01/07 \\ Versão reformulada recebida em 21/06/07 \\ Publicado em 31/07/07
}

${ }^{I}$ Dept. de Zoologia e Botânica, Universidade Estadual Paulista - UNESP,

Campus de São José do Rio Preto, Rua Cristóvão Colombo, 2265,

CEP 15054-000, São José do Rio Preto, SP, Brasil

${ }^{2}$ Programa de Pós-Graduação em Biologia Animal,

Universidade Estadual Paulista - UNESP, Campus de São José do Rio Preto, SP, Brasil

${ }^{3}$ Autor para correspondência: Reinaldo José Fazzio Feres, e-mail: reinaldo@ibilce.unesp.br

\begin{abstract}
Feres, R.J.F., Buosi, R., Daud, R.D. \& Demite, P.R. Ecological patterns of the mite community on euphorbiaceous plants from a Semidecidual forest remnant in the State of São Paulo. Biota Neotrop. May/Aug 2007 vol. 7, no. 2. http://www.biotaneotropica.org.br/v7n2/pt/abstract?article+bn04907022007. ISSN 1676-0603.

The diversity and seasonality of mite community on three indigenous euphorbiaceous plants from a Semidecidual forest remnant were studied by means of monthly samplings performed from May 2003 to April 2004. Diversity, dominance and equitability indexes were applied in order to verify community ecological patterns, and the Pearson correlation coefficient was used to relate mite abundance with the rainfall. About 11,515 mites belonging to 111 species of 73 genera in 25 families were recorded. Among these species, $44 \%$ of them were generalists, while 21 and 35\% are phytophagous and predatory, respectively. The phytophagous mites were the most abundant, performing $45.7 \%$ of mites collected, followed by the predatory and generalist species, which represented 40.5 and $13.7 \%$ of material sampled, respectively. The Phytoseiidae family showed the highest species richness (23), followed by Tarsonemidae (18) and Tydeidae (16). The three euphorbiaceous plants studied showed great species diversity, as the results of indexes were higher than $50 \%$ of theoretical maximum diversity. The species-abundance patterns determined for mite community was equally represented by geometric and log series models. Out of three plant species studied, only Acalypha diversifolia showed potential usage in Integrated Pest Management programs.
\end{abstract}

Keywords: Acari, diversity, Euphorbiaceae, integrated pest management, seasonality.

Resumo

Feres, R.J.F., Buosi, R., Daud, R.D. \& Demite, P.R. Padrões ecológicos da comunidade de ácaros em euforbiáceas de um fragmento de mata Estacional Semidecidual, no Estado de São Paulo. Biota Neotrop. May/Aug 2007 vol. 7, no. 2. http://www.biotaneotropica.org.br/v7n2/pt/abstract?article+bn04907022007. ISSN 1676-0603.

Foi estudada a diversidade e a sazonalidade da comunidade de ácaros em três euforbiáceas nativas de um fragmento de mata estacional semidecidual, através de coletas mensais realizadas de maio de 2003 a abril de 2004. Foram aplicados índices descritores de diversidade, dominância e de equitatibilidade, para estudar os padrões ecológicos da comunidade, e a correlação linear de Pearson, para relacionar a abundância dos ácaros com a pluviosidade. Foram registrados 11.515 ácaros pertencentes a 111 espécies de 73 gêneros e 25 famílias. Dessas espécies, $44 \%$ são generalistas enquanto que 21 e $35 \%$ são fitófagas e predadoras, respectivamente. As fitófagas foram as mais abundantes, representando $45,7 \%$ do material coletado, seguido pelas predadoras e generalistas, que constituíram 40,5 e 13,7\% dos espécimes amostrados, respectivamente. Phytoseiidae foi a família com a maior riqueza de espécies (23), seguida por Tarsonemidae (18) e Tydeidae (16). As três euforbiáceas estudadas apresentaram grande diversidade de espécies, pois os índices calculados foram superiores a $50 \%$ da diversidade máxima teórica prevista. O padrão de abundância da comunidade foi igualmente descrito pelos padrões geométrico e logarítmico. Das três espécies de plantas estudadas, apenas Acalypha diversifolia apresentou potencial para utilização em programas de Manejo Integrado de Pragas.

Palavras-chave: Acari, diversidade, Euphorbiaceae, manejo integrado de pragas, sazonalidade. 


\section{Introdução}

A maioria dos estudos em Acarologia é realizada em agroecossistemas (Walter \& Proctor 1998), porém, pesquisas em áreas florestais podem levar a descoberta de inimigos naturais que apresentem características desejáveis para programas de controle biológico e de manejo integrado de pragas (MIP). Segundo Altieri et al. (2003), plantas nativas podem também servir como reservatórios de inimigos naturais em períodos de escassez de presas, pois esses podem utilizar seu pólen e néctar como alimento, além de certas características morfológicas da planta servirem como abrigo e sítio de oviposição para essas espécies.

Como conseqüência da expansão das atividades agrícolas, muitas áreas florestais foram reduzidas a pequenos fragmentos de mata. Alguns estudos sobre a comunidade de ácaros em fragmentos florestais brasileiros enfatizaram o inventário de espécies. Feres \& Moraes (1998), Feres et al. (2005) e Buosi et al. (2006), relacionaram diversas espécies de ácaros associadas a plantas localizadas em fragmentos de mata estacional semidecidual, na região sudeste do Brasil. Já os padrões ecológicos e a diversidade de espécies na comunidade de ácaros plantícolas de áreas naturais foram verificados por Arruda-Filho \& Moraes (2002), Zacarias \& Moraes (2002), Daud \& Feres (2005) e Lofego \& Moraes (2006). Porém, os padrões ecológicos e a dinâmica populacional da comunidade de ácaros em plantas de fragmentos florestais são ainda pouco conhecidos no Brasil, embora sejam considerados conhecimentos básicos importantes para a realização de programas bem sucedidos de MIP. O objetivo do presente trabalho foi descrever os padrões de diversidade e a dinâmica populacional da comunidade de ácaros de três euforbiáceas nativas em fragmento de mata na Estação Ecológica de Paulo de Faria, SP. Essa Estação Ecológica é considerada atualmente um dos últimos remanescentes da floresta original que recobria a região norte do estado de São Paulo, a de maior índice de desmatamento e com a menor concentração de unidades de conservação do estado (Kronka et al. 1993).

\section{Material e Métodos}

Área de estudo. A Estação Ecológica de Paulo de Faria (19 55' S e $\left.49^{\circ} 31^{\prime} \mathrm{W}\right)$, localizada no município de Paulo de Faria, SP, abrange uma área de 435,73 ha de floresta estacional semidecidual. Segundo a classificação de Köppen, o tipo climático da região é o Cwa-Aw, considerado como tropical quente e úmido, com chuvas de verão e estiagem de inverno (Barcha \& Arid, 1971).

Amostragem. Nesse fragmento florestal foram avaliadas, em coletas mensais realizadas de maio de 2003 a abril de 2004, três espécies de euforbiáceas nativas: Actinostemon communis (Müll. Arg.) e Acalypha diversifolia Jacq., de porte arbustivo e mais abundantes nessa Estação (Stranghetti \& Ranga 1998), e Alchornea glandulosa Poepp. \& Endl, de porte arbóreo, com exemplares localizados na margem da estrada que circunda o fragmento.

Em período anterior ao início das coletas, foram selecionadas e marcadas com placas metálicas numeradas, cinco exemplares de A. glandulosa e dez de A. diversifolia e A. communis, levando-se em consideração a diferença do tamanho relativo das folhas dessas plantas. Foram amostradas 20 folhas de cada planta marcada até uma altura de cerca de $12 \mathrm{~m}$, com auxilio de podão com cabo telescópico. O material coletado foi transportado para o laboratório de Acarologia da UNESP, São José do Rio Preto, SP, acondicionado em sacos de papel envolvidos por sacos plásticos etiquetados, armazenados em caixas isotérmicas de poliestireno com Gelo- $\mathrm{X}^{\circledR} \mathrm{em}$ seu interior, com temperatura variando de 15 à $20^{\circ} \mathrm{C}$.

Os ácaros presentes nas folhas amostradas foram triados sob microscópio estereoscópico e todos os exemplares encontrados, com exceção de Oribatida, foram montados em lâminas de microscopia com meio de Hoyer (Flechtmann 1975, Jeppson et al. 1975), para posterior identificação e contagem sob microscópio óptico com contraste de fases. Os ácaros oribatídeos foram triados e armazenados em álcool etílico a 70\% e posteriormente clarificados em ácido lático para observação em lâmina escavada (Travé et al. 1996).

Exemplares de referência foram depositados na coleção científica de Acari (DZSJRP) - http://splink.cria.org.br, do Departamento de Zoologia e Botânica, Universidade Estadual Paulista (UNESP), São José do Rio Preto, São Paulo.

Análises ecológicas e estatísticas. A diversidade de espécies na comunidade de ácaros foi calculada pelos índices de Shannon-Wiener (H') e Simpson (1-D) (Odum 1988, Krebs 1999). A equitatibilidade (E) e a diversidade máxima teórica (Hmax) foram determinadas de acordo com Krebs (1999), e a curva do coletor segundo Brower \& Zar (1984). A existência de dominância na abundância de espécies na comunidade foi verificada por análise gráfica, através da curva do componente dominância de diversidade e pelo índice de dominância de Simpson (Magurran 1988, Krebs 1999). O ajustamento da curva e os cálculos dos índices ecológicos foram realizados no software PAST versão 1.36 (Hammer et al. 2001).

Para as análises estatísticas, a comunidade de ácaros foi organizada em três grupos, baseando-se no hábito alimentar das espécies pertencentes a cada um: 1) Predadoras, constituído por espécies predadoras e preponderantemente predadoras, 2) fitófagas e 3) generalistas. Nesse último, foram agrupadas as espécies micófagas e de hábito alimentar desconhecido. A abundância das espécies nos diferentes grupos, em cada planta, foi comparada através do teste G. Correções de Bonferroni $(\alpha / n)$ foram usadas para comparações pareadas (Zar 1999). A correlação linear de Pearson (Zar 1999) foi utilizada para estudar possível relação entre abundância da acarofauna com a precipitação mensal, sendo a existência de correlação verificada ao nível de alfa $=0,05$. Para a realização dessa última análise, os dados foram antes transformados pela equação logarítmica Ln (y). Os índices pluviométricos foram obtidos junto à Coordenadoria de Assistência Técnica e Integral (CATI) de Paulo de Faria, SP.

\section{Resultados}

Foram registrados 11.515 ácaros pertencentes a 111 espécies de 73 gêneros e 25 famílias, associados às três espécies de euforbiáceas nativas estudadas. Dentre as espécies acarinas amostradas, 33 foram comuns aos três hospedeiros (Tabela 1).

As generalistas representaram $44 \%$ do total de espécies amostradas, enquanto que 21 e $35 \%$ são fitófagas e predadoras, respectivamente. Por outro lado, as fitófagas representaram $45,7 \%$ do material coletado, quando considerada a abundância dos ácaros nos diferentes grupos. Já as predadoras e as generalistas constituíram 40,5 e 13,7\% dos espécimes amostrados, respectivamente.

Em A. diversifolia $(\mathrm{G}=1.125,4, \mathrm{gl}=2, P<0,001)$ e A. communis $(\mathrm{G}=117,9, \mathrm{gl}=2, P<0,001)$, a abundância das predadoras foi significativamente superior em relação às demais espécies, enquanto que em A. glandulosa, as fitófagas foram as mais numerosas $(\mathrm{G}=2.746,3$, $\mathrm{gl}=2, P<0,001)$. Não houve diferenças significativas no número total de fitófagas e generalistas em A. communis (Figura 1).

Composição geral das espécies. A família com a maior riqueza foi Phytoseiidae, com 23 espécies, seguida por Tarsonemidae (18) e Tydeidae (16). Dez espécies pertencentes a família Phytoseiidae foram comuns nos três hospedeiros, enquanto que Tydeidae e Tarsonemidae apresentaram oito e cinco espécies compartilhadas entre as plantas estudadas, respectivamente (Tabela 1). Em A. diversifolia, Phytoseiidae foi a família com maior número de espécies (19) e de indivíduos (1.308). Já em A. communis, as famílias com as maiores 
Tabela 1. Abundância das espécies de ácaros registradas nas três Euphorbiaceae estudadas de maio de 2003 a abril de 2004 , na "Estação Ecológica de Paulo de Faria", SP.

Table 1. Abundance of mites registered in three Euphorbiaceae from May 2003 to April 2004, in the "Estação Ecológica de Paulo de Faria", SP.

\begin{tabular}{|c|c|c|c|c|c|}
\hline Família & Gênero - espécie & A. diversifolia & A. communis & A. glandulosa & Total \\
\hline \multirow[t]{2}{*}{ Acaridae } & Caloglyphus sp. ${ }^{\mathrm{G}}$ & 103 & 19 & 173 & 295 \\
\hline & Tyrophagus putrescentiae $^{\mathrm{G}}$ & 1 & 0 & 0 & 1 \\
\hline \multirow[t]{3}{*}{ Ascidae } & Asca sp. ${ }^{\mathrm{P}}$ & 270 & 47 & 42 & 359 \\
\hline & Lasioseius sp. ${ }^{\mathrm{P}}$ & 1 & 0 & 0 & 1 \\
\hline & Zercoseius sp. ${ }^{\mathrm{P}}$ & 1 & 0 & 0 & 1 \\
\hline \multirow[t]{2}{*}{ Bdellidae } & Cyta sp. ${ }^{\mathrm{P}}$ & 1 & 0 & 2 & 3 \\
\hline & Hexabdella aff. singula ${ }^{\mathrm{P}}$ & 1 & 0 & 1 & 2 \\
\hline Camerobiidae & Neophyllobius sp. ${ }^{\mathrm{G}}$ & 0 & 1 & 0 & 1 \\
\hline \multirow[t]{3}{*}{ Cheyletidae } & Cheletogenes ornatus ${ }^{\mathrm{P}}$ & 0 & 0 & 2 & 2 \\
\hline & Cheletomimus wellsi $^{\mathrm{P}}$ & 8 & 4 & 13 & 25 \\
\hline & Chiapacheyllus sp. ${ }^{\mathrm{P}}$ & 3 & 3 & 0 & 6 \\
\hline \multirow[t]{3}{*}{ Cunaxidae } & Armascirus sp. ${ }^{\mathrm{P}}$ & 3 & 1 & 5 & 9 \\
\hline & Cunaxoides sp. ${ }^{\mathrm{P}}$ & 1 & 1 & 14 & 16 \\
\hline & Scirula sp. ${ }^{\mathrm{P}}$ & 7 & 0 & 22 & 29 \\
\hline Diptilomiopidae & Diptilomiopus sp. ${ }^{\mathrm{F}}$ & 0 & 0 & 442 & 442 \\
\hline \multirow[t]{5}{*}{ Eriophyidae } & Aceria sp. ${ }^{\mathrm{F}}$ & 15 & 0 & 0 & 15 \\
\hline & Phyllocoptes sp. ${ }^{\mathrm{F}}$ & 0 & 0 & 1723 & 1723 \\
\hline & Procalacarus sp. ${ }^{\mathrm{F}}$ & 0 & 0 & 23 & 23 \\
\hline & Tegonotus sp. ${ }^{\mathrm{F}}$ & 0 & 0 & 1 & 1 \\
\hline & Aff. Tetraspinus sp. ${ }^{\mathrm{F}}$ & 0 & 0 & 832 & 832 \\
\hline \multirow[t]{2}{*}{ Eupalopsellidae } & Aff. Eupalopsis sp. ${ }^{\mathrm{G}}$ & 1 & 0 & 0 & 1 \\
\hline & Exothorhis sp. ${ }^{\mathrm{G}}$ & 0 & 0 & 4 & 4 \\
\hline Eupodidae & Eupodes sp. ${ }^{\mathrm{G}}$ & 9 & 2 & 0 & 11 \\
\hline \multirow[t]{23}{*}{ Phytoseiidae } & Amblydromalus manihoti $^{\mathrm{P}}$ & 0 & 0 & 14 & 14 \\
\hline & Amblyseius aerialis ${ }^{\mathrm{P}}$ & 69 & 17 & 6 & 92 \\
\hline & Amblyseius paulofariensis ${ }^{\mathrm{P}}$ & 465 & 154 & 29 & 648 \\
\hline & Amblyseius chiapensis $^{\mathrm{P}}$ & 87 & 8 & 255 & 350 \\
\hline & Amblyseius herbicolus ${ }^{\mathrm{P}}$ & 30 & 58 & 29 & 117 \\
\hline & Euseius alatus ${ }^{\mathrm{P}}$ & 4 & 0 & 7 & 11 \\
\hline & Euseius citrifolius $^{\mathrm{P}}$ & 8 & 6 & 12 & 26 \\
\hline & Euseius concordis ${ }^{\mathrm{P}}$ & 340 & 94 & 736 & 1170 \\
\hline & Euseius sibelius $^{\mathrm{P}}$ & 0 & 0 & 4 & 4 \\
\hline & Galendromus annectens ${ }^{\mathrm{P}}$ & 24 & 1 & 10 & 35 \\
\hline & Iphiseiodes zuluagai $^{\mathrm{P}}$ & 127 & 4 & 397 & 528 \\
\hline & Metaseiulus adjacentis ${ }^{\mathrm{P}}$ & 16 & 0 & 2 & 18 \\
\hline & Neoseiulus tunus ${ }^{\mathrm{P}}$ & 107 & 10 & 34 & 151 \\
\hline & Paraphytoseius orientalis ${ }^{\mathrm{P}}$ & 0 & 0 & 2 & 2 \\
\hline & Phytoseius cf. plumifer ${ }^{\mathrm{P}}$ & 5 & 0 & 1 & 6 \\
\hline & Proprioseiopsis dominigos ${ }^{\mathrm{P}}$ & 1 & 0 & 1 & 2 \\
\hline & Proprioseiopsis neotropicus ${ }^{\mathrm{P}}$ & 6 & 1 & 8 & 15 \\
\hline & Proprioseiopsis ovatus ${ }^{\mathrm{P}}$ & 3 & 0 & 0 & 3 \\
\hline & Silvaseius cf. barretoae ${ }^{\mathrm{P}}$ & 1 & 0 & 0 & 1 \\
\hline & Transeius bellottii $^{\mathrm{P}}$ & 11 & 0 & 0 & 11 \\
\hline & Typhlodromalus aripo ${ }^{\mathrm{P}}$ & 3 & 0 & 13 & 16 \\
\hline & Typhlodromalus peregrinus ${ }^{\mathrm{P}}$ & 1 & 0 & 1 & 2 \\
\hline & Typhlodromalus sp. ${ }^{\mathrm{P}}$ & 0 & 0 & 2 & 2 \\
\hline \multirow[t]{2}{*}{ Stigmaeidae } & Agistemus sp. ${ }^{\mathrm{P}}$ & 151 & 11 & 31 & 193 \\
\hline & Zetzellia quasagistemas ${ }^{\mathrm{P}}$ & 0 & 0 & 3 & 3 \\
\hline Tarsonemidae & Daidalotarsonemus tesselatus $^{\mathrm{G}}$ & 6 & 2 & 96 & 104 \\
\hline
\end{tabular}


Tabela 1. Continuação...

\begin{tabular}{|c|c|c|c|c|c|}
\hline Família & Gênero - espécie & A. diversifolia & A. communis & A. glandulosa & Total \\
\hline & Daidalotarsonemus sp. ${ }^{\mathrm{G}}$ & 0 & 0 & 2 & 2 \\
\hline & Fungitarsonemus sp1. ${ }^{\mathrm{G}}$ & 46 & 133 & 109 & 288 \\
\hline & Fungitarsonemus sp2. ${ }^{\mathrm{G}}$ & 2 & 0 & 5 & 7 \\
\hline & Metatarsonemus megasolenidii ${ }^{\mathrm{G}}$ & 30 & 27 & 99 & 156 \\
\hline & Metatarsonemus sp. ${ }^{\mathrm{G}}$ & 0 & 1 & 0 & 1 \\
\hline & Tarsonemus (Floridotarsonemus) sp. ${ }^{\mathrm{G}}$ & 0 & 5 & 0 & 5 \\
\hline & Tarsonemus (Tarsonemus) confusus ${ }^{\mathrm{G}}$ & 10 & 4 & 6 & 20 \\
\hline & Tarsonemus (Tarsonemus) sp. $1^{\mathrm{G}}$ & 1 & 0 & 0 & 1 \\
\hline & Tarsonemus (Tarsonemus) sp. $2^{\mathrm{G}}$ & 0 & 1 & 0 & 1 \\
\hline & Tarsonemus (Tarsonemus) sp. $3^{\mathrm{G}}$ & 0 & 9 & 4 & 13 \\
\hline & Tarsonemus (Tarsonemus) sp. $4^{\mathrm{G}}$ & 1 & 0 & 0 & 1 \\
\hline & Tarsonemus (Tarsonemus) sp. $5^{\mathrm{G}}$ & 0 & 0 & 1 & 1 \\
\hline & Tarsonemus (Tarsonemus) sp. $6^{\mathrm{G}}$ & 0 & 0 & 1 & 1 \\
\hline & Xenotarsonemus sp. $1^{\mathrm{G}}$ & 12 & 4 & 2 & 18 \\
\hline & Xenotarsonemus sp. $2^{\mathrm{G}}$ & 0 & 3 & 2 & 5 \\
\hline & Xenotarsonemus sp. $3^{\mathrm{G}}$ & 2 & 0 & 0 & 2 \\
\hline & Xenotarsonemus sp. $4^{\mathrm{G}}$ & 0 & 3 & 0 & 3 \\
\hline \multirow[t]{5}{*}{ Tenuipalpidae } & Brevipalpus phoenicis ${ }^{\mathrm{F}}$ & 2 & 3 & 53 & 58 \\
\hline & Tenuipalpus aff. anoplomexus ${ }^{\mathrm{F}}$ & 2 & 0 & 0 & 2 \\
\hline & Tenuipalpus aff. heteropyxis ${ }^{\mathrm{F}}$ & 0 & 0 & 790 & 790 \\
\hline & Tenuipalpus aff. unimerus ${ }^{\mathrm{F}}$ & 1 & 0 & 0 & 1 \\
\hline & Tenuipalpus aff. zanthus ${ }^{\mathrm{F}}$ & 1 & 0 & 0 & 1 \\
\hline \multirow[t]{7}{*}{ Tetranychidae } & Aponychus aff. schultzi ${ }^{\mathrm{F}}$ & 503 & 0 & 1 & 504 \\
\hline & Atrichoproctus uncinatus ${ }^{\mathrm{F}}$ & 0 & 0 & 2 & 2 \\
\hline & Eotetranychus sp. ${ }^{\mathrm{F}}$ & 1 & 0 & 0 & 1 \\
\hline & Neotretranychus asper ${ }^{\mathrm{F}}$ & 2 & 0 & 0 & 2 \\
\hline & Oligonychus gossypii ${ }^{\mathrm{F}}$ & 0 & 1 & 0 & 1 \\
\hline & Tetranychus mexicanus ${ }^{\mathrm{F}}$ & 86 & 0 & 28 & 114 \\
\hline & Tetranychus riopretensis ${ }^{\mathrm{F}}$ & 0 & 156 & 0 & 156 \\
\hline \multirow[t]{16}{*}{ Tydeidae } & Homeopronematus sp. ${ }^{\mathrm{P}}$ & 113 & 196 & 188 & 497 \\
\hline & Lorryia formosa ${ }^{\mathrm{F}}$ & 18 & 6 & 23 & 47 \\
\hline & Lorryia sp. $1^{\mathrm{F}}$ & 45 & 195 & 204 & 444 \\
\hline & Lorryia sp. $2^{\mathrm{F}}$ & 0 & 2 & 0 & 2 \\
\hline & Lorryia sp. $3^{\mathrm{F}}$ & 3 & 0 & 0 & 3 \\
\hline & Lorryia sp. $4^{\mathrm{F}}$ & 1 & 0 & 0 & 1 \\
\hline & Meyerellinae sp. ${ }^{\mathrm{G}}$ & 3 & 9 & 4 & 16 \\
\hline & Neolorryia boycei ${ }^{\mathrm{G}}$ & 4 & 12 & 4 & 20 \\
\hline & Paralorryia sp. ${ }^{\mathrm{G}}$ & 1 & 0 & 0 & 1 \\
\hline & Parapronematus sp. ${ }^{\mathrm{P}}$ & 14 & 61 & 37 & 112 \\
\hline & Pausia sp. ${ }^{\mathrm{G}}$ & 0 & 1 & 0 & 1 \\
\hline & Pretydeus sp. ${ }^{\mathrm{G}}$ & 0 & 0 & 2 & 2 \\
\hline & Pronematus sp. ${ }^{\mathrm{P}}$ & 49 & 49 & 81 & 179 \\
\hline & Pseudolorryia sp. ${ }^{\mathrm{G}}$ & 2 & 1 & 0 & 3 \\
\hline & Triophtydeus sp. ${ }^{\mathrm{G}}$ & 14 & 56 & 16 & 86 \\
\hline & Tydeus sp. ${ }^{\mathrm{G}}$ & 1 & 0 & 0 & 1 \\
\hline \multirow[t]{2}{*}{ Winterschmidtiidae } & Czenspinskia sp. ${ }^{\mathrm{G}}$ & 123 & 40 & 301 & 464 \\
\hline & Oulenzia sp. ${ }^{\mathrm{G}}$ & 13 & 0 & 7 & 20 \\
\hline Ceratozetidae & Ceratozetes cf. catarinensis ${ }^{\mathrm{G}}$ & 70 & 0 & & 70 \\
\hline \multirow[t]{2}{*}{ Cymbaeremaeidae } & Scapheremaeus sp. $1^{\mathrm{G}}$ & 1 & 2 & 0 & 3 \\
\hline & Scapheremaeus sp. $2^{\mathrm{G}}$ & 2 & 2 & 0 & 4 \\
\hline Galumnidae & Galumna sp. $1^{\mathrm{G}}$ & 9 & 5 & 2 & 16 \\
\hline
\end{tabular}


Tabela 1. Continuação...

\begin{tabular}{|c|c|c|c|c|c|}
\hline Família & Gênero - espécie & A. diversifolia & A. communis & A. glandulosa & Total \\
\hline & Galumna sp.2 ${ }^{\mathrm{G}}$ & 1 & 1 & 0 & 2 \\
\hline Mochlozetidae & Dynatozetes cf. amplus ${ }^{\mathrm{G}}$ & 0 & 0 & 3 & 3 \\
\hline \multirow[t]{2}{*}{ Oripodidae } & Oripoda sp. $1^{\mathrm{G}}$ & 3 & 3 & 4 & 10 \\
\hline & Oripoda sp. $2^{\mathrm{G}}$ & 5 & 4 & 0 & 9 \\
\hline \multirow[t]{2}{*}{ Pheroliodidae } & Pheroliodes sp. $1^{\mathrm{G}}$ & 1 & 0 & 0 & 1 \\
\hline & Pheroliodes sp. $2^{\mathrm{G}}$ & 0 & 1 & 0 & 1 \\
\hline \multirow[t]{4}{*}{ Scheloribatidae } & Hemileius cf. initialis ${ }^{\mathrm{G}}$ & 2 & 0 & 0 & 2 \\
\hline & Scheloribates sp. $1^{\mathrm{G}}$ & 2 & 0 & 2 & 4 \\
\hline & Scheloribates sp. $2^{\mathrm{G}}$ & 1 & 0 & 0 & 1 \\
\hline & Scheloribates sp. $3^{\mathrm{G}}$ & 3 & 3 & 0 & 6 \\
\hline Scutoverticidae & Arthrovertex cf. hauseri ${ }^{\mathrm{G}}$ & 1 & 0 & 0 & 1 \\
\hline Abundância & & 3097 & 1443 & 6975 & 11515 \\
\hline Riqueza & & 80 & 53 & 68 & 111 \\
\hline
\end{tabular}

(P) Predadores e preponderantemente predadores (Hernandes \& Feres 2005, Gerson et al. 2003, McMurtry \& Croft 1997, Krantz 1978, Flechtmann 1975), (F) Fitófagos (Hernandes \& Feres 2006, Badii et al. 2001, Lindquist et al. 1996, Jeppson et al. 1975, Flechtmann 1973), (G) Generalistas (Lindquist 1986, Krantz 1978, Gerson 1968, Smirnoff 1957, Baker \& Wharton 1952).

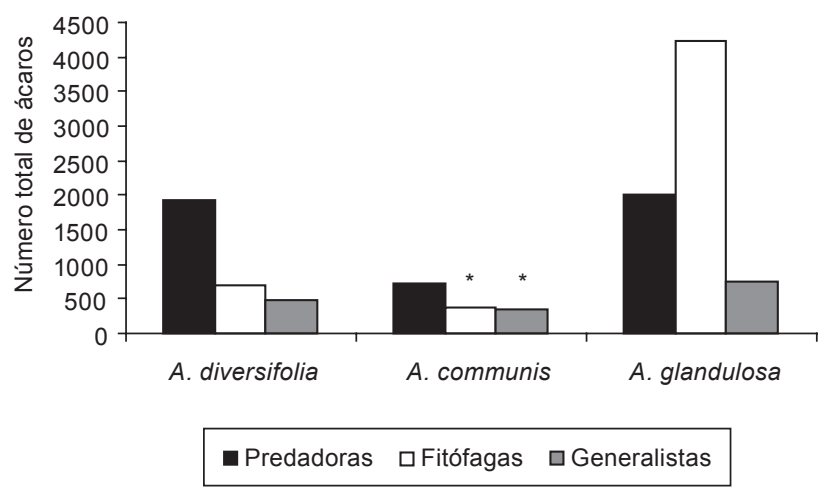

Figura 1. Número total de espécies predadoras, fitófagas e generalistas nas três euforbiáceas nativas estudadas, no período de maio de 2003 a abril de 2004, na "Estação Ecológica de Paulo de Faria", SP. *Não diferem significativamente ao nível de $5 \%$ de probabilidade pelo teste $\mathrm{G}$.

Figure 1. Total number of predatory, phytophagous and generalist species on three indigenous euphorbiaceous plants studied from May 2003 to April 2004 in the "Estação Ecológica de Paulo de Faria", SP. *No statistical differences by G-test $(\mathrm{p}<0.05)$.

riqueza e abundância foram Tarsonemidae e Tydeidae, enquanto em A. glandulosa foram Phytoseiidae e Eriophyidae, respectivamente.

As espécies mais abundantes foram Phyllocoptes sp. (Eriophyidae), Euseius concordis (Chant) (Phytoseiidae), Aff. Tetraspinus (Eriophyidae), Tenuipalpus aff. heteropyxis (Tenuipalpidae) e Amblyseius paulofariensis Demite, Lofego \& Feres (Phytoseiidae), sendo a segunda e a última espécies predadoras e as demais fitófagas. Euseius concordis e A. paulofariensis foram comuns às três euforbiáceas. Por outro lado, Phyllocoptes sp., Aff. Tetraspinus sp. e $T$. aff. heteropyxis foram encontrados somente em A. glandulosa (Tabela 1).

A espécie mais abundante em A. diversifolia foi a fitófaga Aponychus aff. schultzi (Tetranychidae) com 503 indivíduos, seguida pelas predadoras A. paulofariensis (465) e E. concordis (340). Já em A. communis, as espécies mais abundantes foram a predadora Homeopronematus sp. (Tydeidae) (196), e as fitófagas Lorryia sp. 1. (Tydeidae) (195) e Tetranychus riopretensis Feres \& Flechtmann (Tetranychidae) (156), sendo essa última encontrada somente nesse hospedeiro. Em A. glandulosa, a espécie dominante na comunidade de ácaros foi Phyllocoptes sp. com 1.723 indivíduos, seguida por Aff. Tetraspinus sp. (832) e T. aff. heteropyxis (790). Nesse hospedeiro, as predadoras mais abundantes foram os fitoseídeos $E$. concordis (736), Iphiseiodes zuluagai Denmark \& Muma (397) e Amblyseius chiapensis DeLeon (255), espécies que também foram comuns nas outras euforbiáceas estudadas (Tabela 1).

Padrões de diversidade. Em A. diversifolia e A. glandulosa foi registrada a maior acumulação de novos registros de espécies ao longo das coletas em relação a $A$. communis (Figura 2). Nesse último hospedeiro, a curva do coletor atingiu a assíntota mais cedo em relação aos demais (agosto/2003), com acréscimos de algumas espécies raras em dezembro e nas três últimas coletas (fevereiro a abril/2004). Por outro lado, a assíntota da curva em A. glandulosa foi alcançada somente em fevereiro, enquanto que em A. diversifolia em novembro. Nesse último, houve acréscimo de algumas espécies nas coletas finais, porém, essas foram encontradas em pequeno número (Figura 2).

A euforbiácea que apresentou maior abundância de ácaros foi A. glandulosa, com 6.975 indivíduos amostrados, enquanto que A. diversifolia e A. communis apresentaram 2.995 e 1.443 indivíduos, respectivamente. Por outro lado, foi registrada a maior diversidade de espécies na comunidade de ácaros em A. diversifolia e A. communis, pois, ambas apresentaram os maiores valores nos dois índices de diversidade aplicados (Shannon-Wiener e Simpson). A diversidade de ácaros encontrada nas três espécies vegetais estudadas foi superior a 50\% da diversidade máxima teórica prevista (Tabela 2).

Embora tenha apresentado a menor riqueza de espécies (53), A. communis apresentou a maior equitatibilidade em relação às outras duas plantas. Em A. diversifolia e A. glandulosa foram registradas 80 e 68 espécies e a equitatibilidade encontrada foi de 0,67 e 0,63, respectivamente (Tabela 2 ).

As curvas do componente dominância, determinadas para as três plantas estudadas, apresentaram inclinações semelhantes e 
Tabela 2. Parâmetros ecológicos da comunidade de ácaros das três Euphorbiaceae estudadas, no fragmento de mata da "Estação Ecológica de Paulo de Faria", SP. Table 2. Ecological parameters of mite community on three Euphorbiaceae, in the forest fragment of the "Estação Ecológica de Paulo de Faria", SP.

\begin{tabular}{lccc}
\hline \multicolumn{1}{c}{ Parâmetros ecológicos } & A. diversifolia & A. communis & A. glandulosa \\
\hline Riqueza (S) & 80 & 53 & 68 \\
Indivíduos (n) & 2.995 & 1.443 & 6.975 \\
Dominância (D) & 0,086 & 0,081 & 0,11 \\
Shannon-Wiener (H') & 2,95 & 2,87 & 2,67 \\
Simpson (1-D) & 0,91 & 0,92 & 0,89 \\
Equitabilidade (J) & 0,67 & 0,72 & 0,63 \\
Diversidade máxima teórica (Hmax) & 4,38 & 3,97 & 4,22 \\
\hline
\end{tabular}

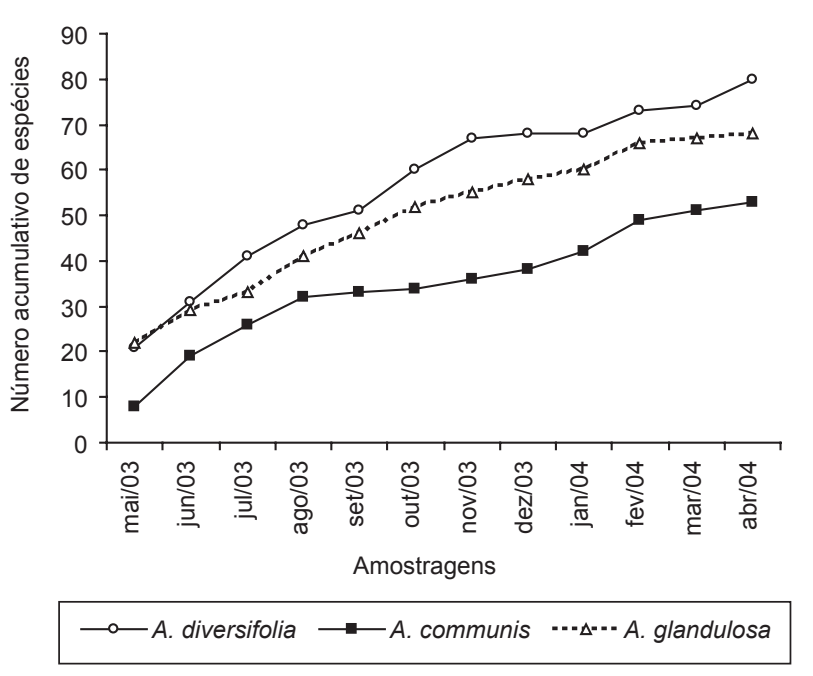

Figura 2. Curva acumuladora de espécies determinada para as três euforbiáceas nativas estudadas, no período de maio de 2003 a abril de 2004, na "Estação Ecológica de Paulo de Faria", SP.

Figure 2. Species-sampled curve performed for three indigenous euphorbiaceous studied, from May 2003 to April 2004, in the "Estação Ecológica de Paulo de Faria", SP.

se ajustaram similarmente aos modelos dos padrões geométrico e logarítmico (Figura 3). Contudo, o maior índice de dominância de espécies na comunidade de ácaros foi encontrado em A. glandulosa $(\mathrm{D}=0,11)$, enquanto que $A$. diversifolia $A$. communis apresentaram dominância (D) de 0,09 e 0,08, respectivamente (Tabela 2).

Dinâmica populacional. Em A. diversifolia, as espécies predadoras foram mais abundantes em relação às demais em todas as amostras, porém, apresentaram maior número de indivíduos durante o período de floração, que ocorreu de junho a novembro, época com baixo índice pluviométrico registrado (Figura 4a). Dentre as predadoras, A. paulofariensis e E. concordis foram as que apresentaram maior abundância durante a floração, e ocorrência de pico populacional em junho e setembro (estação seca), respectivamente. As espécies fitófagas foram frequientes em todas as coletas, com exceção dos meses de maio e novembro, e apresentaram pico populacional em julho (Figura 4a). O padrão de ocorrência e abundância das fitófagas em $A$. diversifolia foi influenciado principalmente por $A$. aff. schultzi. Já as espécies generalistas foram mais abundantes durante a estação chuvosa e apresentaram pico populacional em março (Figura 4a), sendo Caloglyphus sp. (Acaridae), a mais abundante dentre elas nesse período. As predadoras foram correlacionadas negativamente com a pluviosidade nessa euforbiácea $(r=-0,63, p=0,0266)$.
Em A. communis, as espécies predadoras foram freqüentes em todas as coletas realizadas, e apresentaram dois picos populacionais, sendo um no início (novembro) e outro em plena estação chuvosa (fevereiro) (Figura 4b). No primeiro, a predadora mais abundante foi Homeopronematus sp. e no segundo pico, Parapronematus sp. (Tydeidae, Pronematinae). Nessa planta, as espécies fitófagas foram mais abundantes durante a estação chuvosa (Figura 4b), sendo Lorryia sp. 1 e T. riopretensis, as espécies mais numerosas no período. As espécies generalistas foram também mais abundantes durante o período chuvoso e apresentaram pico populacional em fevereiro (Figura 4b), sendo Fungitarsonemus sp.1, a espécie mais abundante. Não foi registrada correlação significativa da acarofauna de $A$. communis com a precipitação mensal.

As espécies predadoras associadas a A. glandulosa foram também freqüentes em todas as coletas realizadas, porém menos abundantes durante o período chuvoso. Essas espécies apresentaram pico populacional em agosto (Figura 4c), sendo E. concordis, a mais abundante dentre elas. Já as espécies fitófagas dessa euforbiácea foram mais abundantes e freqüentes durante a estação chuvosa e apresentaram pico populacional em janeiro (Figura 4c), sendo Phyllocoptes sp. a mais abundante delas. As espécies generalistas em A. glandulosa também apresentaram pico de abundância durante janeiro (Figura 4c), e Czenspinskia sp. (Winterschmidtiidae) foi a espécie mais abundante registrada no período. Nessa árvore, a população de predadoras correlacionou-se negativamente $(r=-0,57, p=0,0493)$, enquanto que a de fitófagas positivamente $(\mathrm{r}=0,75, \mathrm{p}=0,0045)$ com a precipitação mensal registrada.

\section{Discussão}

Composição geral e diversidade da comunidade de ácaros. $\mathrm{O}$ número de taxa coletados evidenciou a grande riqueza de ácaros no fragmento de mata estudado. Das 111 espécies coletadas, foram identificadas 34 espécies nominais. Dos taxa não determinados, pelo menos três gêneros e 68 espécies são provavelmente novas para ciência, o que reforça a necessidade da realização de inventários da acarofauna em áreas naturais. Além da grande riqueza, pode-se observar alta equitatibilidade das espécies dentro da comunidade de ácaros das três euforbiáceas, o que resultou em grandes valores nos índices utilizados para medir a diversidade. Esse padrão tem sido encontrado por outros autores que estudaram a diversidade de ácaros em fragmentos florestais brasileiros: Arruda-Filho \& Moraes (2002) em arecáceas da mata atlântica; Zacarias \& Moraes (2002) em euforbiáceas de fragmentos de mata Atlântica e de floresta estacional semidecidual; e Daud \& Feres (2005) em Mabea fistulifera Mart. (Euphorbiaceae) de dois fragmentos de mata estacional semidecidual. Segundo Huston (1995) esse valor é normalmente encontrado em ambientes com alta heterogeneidade ambiental, tal como o fragmento de mata estudado, diferentemente de monoculturas, ambientes com 


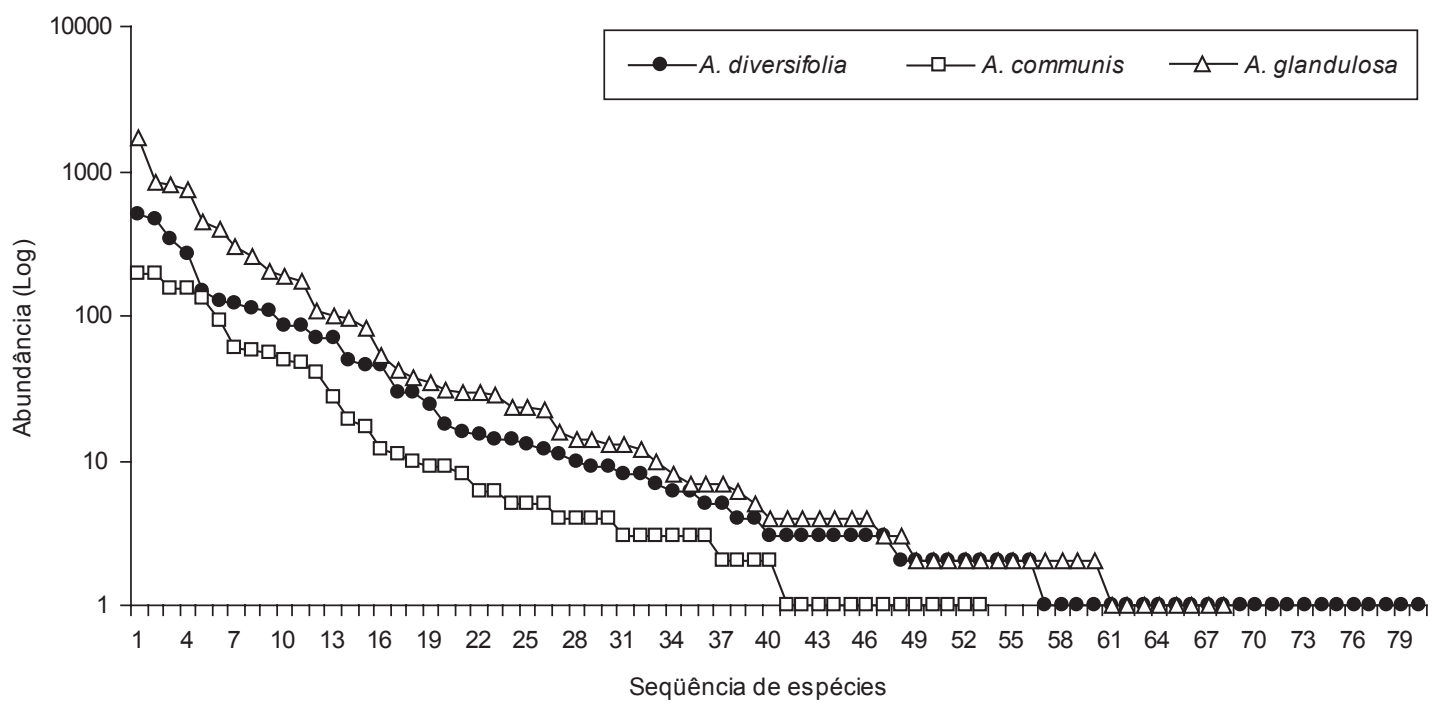

Figura 3. Curva do componente dominância de espécies para a comunidade de ácaros das três euforbiáceas nativas estudadas, no período de maio de 2003 a abril de 2004. Padrão geométrico: A. diversifolia $(\mathrm{k}=0,07, \mathrm{p}<0,001)$, A. communis $(\mathrm{k}=0,09, \mathrm{p}<0,001)$, A. glandulosa $(\mathrm{k}=0,09, \mathrm{p}<0,001)$; Padrão logarítmico: A. diversifolia $(\alpha=15,1, \mathrm{p}<0,001)$, A. communis $(\alpha=10,8, \mathrm{p}<0,001)$, A. glandulosa $(\alpha=10,4, \mathrm{p}<0,001)$.

Figure 3. Species abundance models of mites communities from three indigenous euphorbiaceous studied, from May 2003 to April 2004. The geometric series: A. diversifolia $(\mathrm{k}=0.07, \mathrm{p}<0.001)$, A. communis $(\mathrm{k}=0.09, \mathrm{p}<0.001)$, A. glandulosa $(\mathrm{k}=0.09, \mathrm{p}<0.001)$; The log series model: A. diversifolia $(\alpha=15.1$, $\mathrm{p}<0.001)$, A. communis $(\alpha=10.8, \mathrm{p}<0.001)$, A. glandulosa $(\alpha=10.4, \mathrm{p}<0.001)$.

impacto antrópico acentuado e aqueles com condições abióticas extremas (e.g. desertos, montanhas).

Das 28 espécies nominais registradas em A. glandulosa, somente oito delas foram registradas por Zacarias \& Moraes (2002), no mesmo hospedeiro em três fragmentos de mata localizados na região sudeste do estado de São Paulo. Dentre essas, sete pertencem a família Phytoseiidae e uma a Tydeidae.

Phytoseiidae foi a família que apresentou a maior proporção de espécies compartilhadas entre as plantas estudadas, ao contrário dos fitófagos mais abundantes (Tetranychidae e Eriophyidae), que apresentam maior especificidade de hospedeiro. Várias espécies de fitoseídeos são predadoras de ácaros, insetos e de seus ovos, todavia, podem utilizar outras fontes de alimento, tais como pólen, néctar e secreções açucaradas de afídeos (McMurtry \& Croft 1997, Gerson et al. 2003). Além disso, esses predadores podem utilizar estruturas vegetais como abrigo (e.g. domáceas) e local para oviposição (Romero \& Benson 2004, 2005). Por esses motivos, os fitoseídeos não são específicos ao hospedeiro, utilizando os recursos que ele oferece. Outros autores coletaram algumas das espécies registradas neste trabalho em outras localidades e em diferentes hospedeiros (Zacarias \& Moraes 2002, Feres et al. 2002, Daud \& Feres 2005, Demite \& Feres 2005, 2007). Buosi et al. (2006) também registraram E. concordis e I. zuluagai em oito e seis espécies vegetais, respectivamente, no mesmo fragmento de mata aqui analisado. Apesar de várias espécies pertencentes a família Tetranychidae ocorrerem em diferentes hospedeiros (Bolland et al. 1998), as registradas neste estudo apresentaram especificidade, com exceção de Tetranychus mexicanus (McGregor). Já todos os Eriophyoidea (Eriophyidae e Diptilomiopidae) apresentaram especificidade de hospedeiro (a maioria em A. glandulosa), o que está de acordo com o padrão encontrado por outros autores para este grupo (Lindquist et al. 1996).

As famílias Tydeidae e Tarsonemidae também apresentaram várias espécies comuns às três euforbiáceas. Nessas famílias podem ser encontradas espécies de hábito alimentar variado como fitófago, predador (somente em Tydeidae), micófago, nectarívoro e detritívoro (Lindquist 1986, Gerson et al. 2003). Isso permite que essas espécies explorem diferentes espécies vegetais, com exceção geralmente das fitófagas, e como os fitoseídeos, são relacionadas aos recursos e não ao hospedeiro.

A menor diversidade na comunidade de ácaros em A. glandulosa deve-se a grande abundância de Phyllocoptes sp. aí registrada. A grande infestação dessa espécie influenciou no valor superior do índice de dominância (D) de A. glandulosa em relação às outras duas plantas. Além disso, nesse hospedeiro foi registrada maior abundância de fitófagas, ao contrário de $A$. diversifolia e $A$. communis, que apresentaram número superior de ácaros predadores. Nesse caso, as predadoras registradas nessas duas últimas euforbiáceas, principalmente as predadoras generalistas, podem estar funcionando como reguladores da diversidade, pois, consomem preferencialmente presas que estão em maior número, impedindo que essas se tornem dominantes na comunidade. Segundo Huston (1995), predadores que se alimentam da espécie mais abundante na comunidade podem promover o aumento da diversidade da mesma, caso essa última espécie ocasione um efeito negativo nas populações de nichos ecológicos similares a ela.

Embora tenham sido registradas riqueza inferior e menor número de acumulação de novos registros de espécies ao longo das coletas em relação às outras duas espécies de euforbiáceas, a comunidade de ácaros de A. communis foi a que apresentou a maior equitatibilidade que, no caso, influenciou o alto valor de diversidade aí encontrado (Magurran 1988). Além disso, a maioria das espécies amostradas nas coletas finais em A. diversifolia e A. glandulosa foi pouco abundante, e provavelmente não associadas ao hospedeiro em que foram amostradas. Nesse caso, a curva do coletor não foi um método adequado para medir a biodiversidade de espécies, porém foi eficiente em mostrar que o número de coletas realizadas foi suficiente para representar a acarofauna (Brower \& Zar 1984), pois independente- 

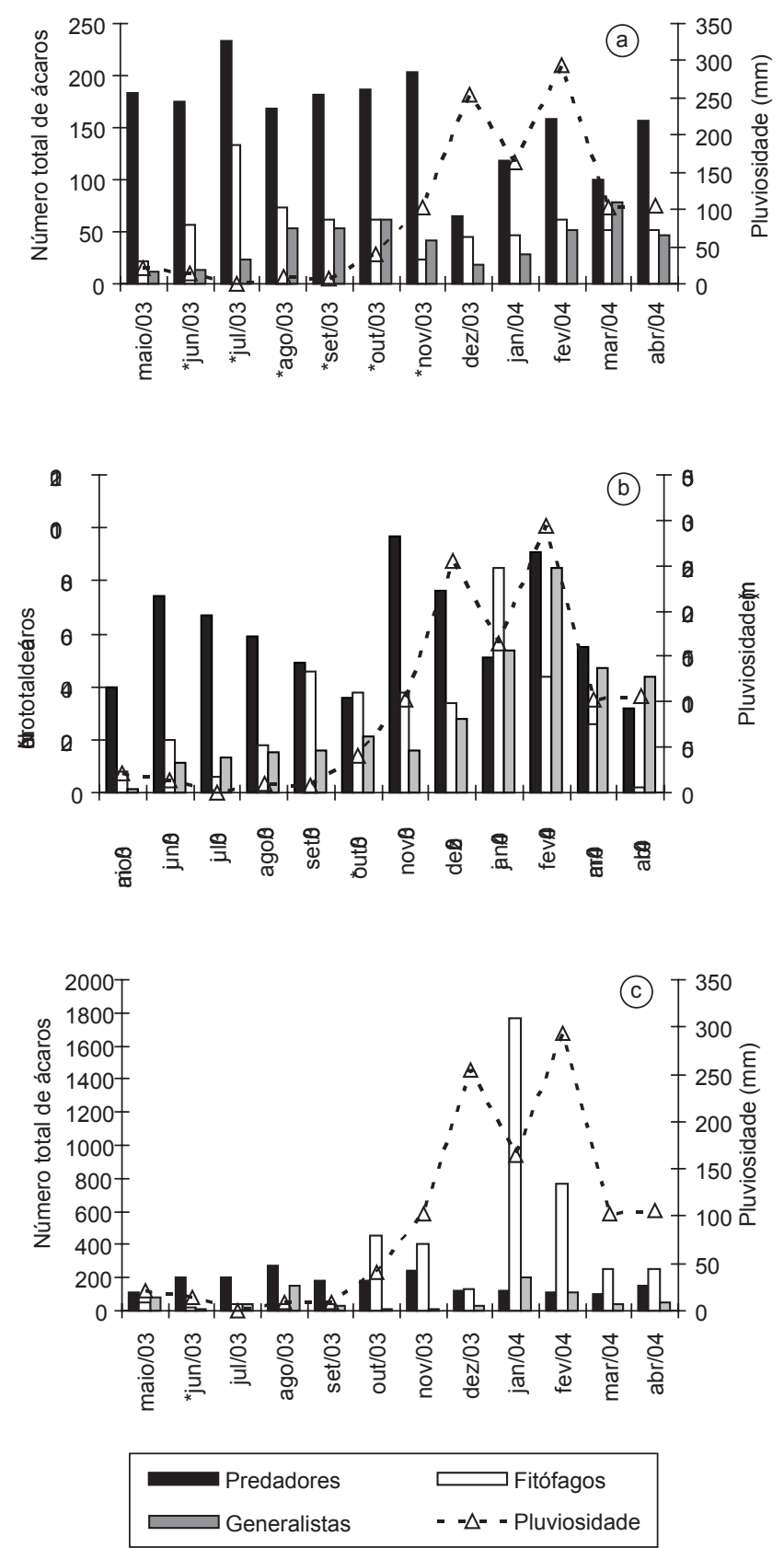

Figura 4. Dinâmica populacional das espécies predadoras, fitófagas e generalistas nas três euforbiáceas e pluviosidade total no período de maio de 2003 a abril de 2004, na "Estação Ecológica de Paulo de Faria", SP. a) A. diversifolia; b) A. communis; e c) A. glandulosa. *Período de floração.

Figure 4. Population dynamics of predatory, phytophagous and generalist species on three euphorbiaceous studied and total rainfall from May 2003 to April 2004 in the "Estação Ecológica de Paulo de Faria", SP. a) A. diversifolia; b) A. communis; and c) A. glandulosa. *Flowering period.

mente do período de amostragem, a assíntota da curva foi atingida nas três espécies vegetais.

O padrão de abundância da acarofauna nas três euforbiáceas foi igualmente representado pelos modelos geométrico e logarítmico, o que está de acordo com May (1975) apud Magurran (1988), que afirma que ambos os modelos são intimamente relacionados. Magurran (1988) também enumera alguns autores que observaram padrões de abundância de algumas comunidades similarmente descritos pelos modelos geométrico e logarítmico. O padrão geométrico é normalmente encontrado em comunidades pobres em número de espécies, tais como as de ambientes poluídos, de condições abióticas extremas e em comunidades em estágio inicial de sucessão ecológica (Gray 1987), diferentemente do fragmento de mata estudado.

Dinâmica populacional. As espécies predadoras registradas em A. diversifolia e A. glandulosa foram freqüentes em todas as coletas, porém mais abundantes durante a estação seca. Dentre essas, as mais representativas foram $E$. concordis nessas duas euforbiáceaes e A. paulofariensis, somente em A. diversifolia. Segundo McMurtry \& Croft (1997), fitoseídeos do gênero Euseius Wainstein são predadores do tipo IV, que se alimentam de presas, mas preferem e se desenvolvem melhor com pólen, enquanto que Amblyseius Berlese são do tipo III, considerados predadores generalistas que podem sobreviver com outras fontes alimentares, inclusive com pólen. O aumento populacional desses predadores em $A$. diversifolia na estação seca pode estar mais correlacionado com a disponibilidade de pólen do que com a menor incidência de chuvas, pois esse também é o período de floração dessa espécie vegetal. Embora o pólen dessa planta não tenha sido testado como alimento para esses ácaros, alguns trabalhos evidenciaram que outras espécies de Amblyseius (Lofego \& Moraes 2005, Kennett et al. 1979) e Euseius (Daud \& Feres 2004, Moraes \& Lima 1983) podem utilizar pólen de algumas espécies vegetais como alimento.

Embora frequientes em todas as coletas, as espécies predadoras em A. communis foram mais abundantes durante a estação chuvosa, período esse, de maior abundância das espécies fitófagas. Isso é sugestivo de que as predadoras podem utilizar essas espécies fitófagas como alimento. Algumas espécies pertencentes ao mesmo gênero das predadoras mais abundantes registradas nessa planta, no caso Homeopronematus Andre e Parapronematus Baker, são consideradas importantes reguladoras de espécies-praga, inclusive de tetraniquídeos (Gerson et al. 2003).

As espécies fitófagas em $A$. diversifolia foram mais abundantes durante a estação seca. O padrão de ocorrência dessas espécies foi influenciado principalmente por $A$. aff. schultzi, fitófaga mais abundante nesse hospedeiro. De acordo com Flechtmann (1981), as infestações de ácaros tetraniquídeos são favorecidas em ambientes secos, o que pode explicar a maior abundância dessa espécie nesse período. Por outro lado, as fitófagas em A. communis e A. glandulosa foram mais numerosas durante a estação chuvosa, inclusive, nesse último hospedeiro, a ocorrência desses ácaros foi correlacionada positivamente com a precipitação mensal. O padrão de ocorrência das fitófagas em A. glandulosa foi influenciada por Phyllocoptes sp., enquanto em A. communis por Lorryia sp. 1. e T. riopretensis. A maior abundancia da última espécie no período chuvoso difere do padrão geral de ocorrência acima mencionado para ácaros tetraniquídeos.

As espécies generalistas foram mais abundantes durante a estação chuvosa nas três euforbiáceas analisadas. As espécies mais representativas nesse período sobre A. diversifolia e A. gladulosa pertencem a subordem Acaridida que possuem, em sua maioria, ácaros de tegumento delgado e, portanto, sensíveis a incidência solar e ambientes secos (Baker \& Wharton 1952). Essa característica também é valida para Fungitarsonemus sp.1 (Lindquist 1986), espécie generalista mais abundante em A. communis no período chuvoso.

Com base nesses resultados, dentre as três plantas estudadas, A. diversifolia foi a que apresentou as melhores características para possível utilização em programas de manejo integrado de pragas, com grande abundância de ácaros predadores e período de floração mais longo (seis meses), o que pode permitir a manutenção de inimigos naturais por maior período. Entretanto, a eficácia de seu pólen como alimento para espécies preponderantemente predadoras e a eficiência dos fitoseídeos registrados como potenciais inimigos naturais de pragas agrícolas deverão ser ainda estudadas. 


\section{Agradecimentos}

À Fundação de Amparo a Pesquisa do estado de São Paulo (FAPESP) pelo apoio financeiro (Processos nº 98/7099-0 e 02/12086-2) e concessão de bolsa ao segundo autor. Ao Dr. Antonio Carlos Lofego (Universidade Estadual Paulista - UNESP, São José do Rio Preto, SP) pelo auxílio na identificação dos Phytoseiidae e Tarsonemidae e ao Dr. Anibal Ramadan Oliveira (Universidade Estadual de Santa Cruz, Ilhéus, BA) pela identificação dos Oribatida. À Profa. Dra. Neusa Taroda Ranga (Universidade Estadual Paulista - UNESP, São José do Rio Preto, SP) pela identificação das plantas estudadas. À Coordenadoria de Assistência Técnica e Integral (CATI) de Paulo de Faria, SP, pelos dados metereológicos cedidos e ao Instituto Florestal por permitir a realização desse trabalho na área da Estação Ecológica e pelo apoio dos funcionários na realização das coletas. "Parte do Programa BIOTA/FAPESP - O Instituto Virtual da Biodiversidade, http://www.biota.org.br

\section{Referências}

ALTIERI, M.A., SILVA, E.N. \& NICHOLLS, C.I. 2003. O papel da biodiversidade no manejo de pragas. Editora Holos, Ribeirão Preto.

ARRUDA-FILHO, G.P. De \& MORAES, G.J. De. 2002. Grupos de ácaros (Arthropoda, Acari) encontrados em Arecaceae da mata Atlântica do estado de São Paulo. Biota Neotrop. 2 (1): http://www.biotaneotropica. org.br/v2n1/pt/abstract?article+BN01502012002

BADII, M.H., FLORES, A.E., PONCE, G., LANDEROS, J. \& QUIROZ, H. 2001. Does Lorryia formosa Cooreman (Acari: Prostigmata: Tydeidae) population visit or reside on citrus foliage? In Acarology: Proceedings of the 10th International Congress (R.B. Halliday, D.E. Walter, H.C. Proctor, R.A. Norton \& M.J. Collof, eds.). CSIRO Publishing, Melbourne. p.413-417.

BAKER, E.W. \& WHARTON, A. E. 1952. An introduction to Acarology. MacMillan Co., New York.

BARCHA, S.F. \& ARID, F.M. 1971. Estudo da evapotranspiração na região norte-ocidental do Estado de São Paulo. Revta. Cient. Fac. Ci. Letr. Votuporanga 1:97-122.

BOLLAND, H.R., GUTIERREZ, J. \& FLECHTMANN, C.H.W. 1998. World catalogue of the spider mite family (Acari: Tetranychidae). Brill, Leiden.

BROWER, J.E. \& ZAR, J.H. 1984. Field \& laboratory methods of General Ecology. 2 ed. Wm. C. Brown Publishers, Dubuque.

BUOSI, R., FERES, R.J.F., OLIVEIRA, A.R., LOFEGO, A.C. \& HERNANDES, F.A. 2006. Ácaros plantícolas (Acari) da "Estação Ecológica de Paulo de Faria”, estado de São Paulo, Brasil. Biota Neotrop. 6 (1): http://www. biotaneotropica.org.br/v6n1/pt/abstract?article+bn02006012006

DAUD, R.D. \& FERES, R.J.F. 2004. O valor de Mabea fistulifera Mart. (Euphorbiaceae), planta nativa do Brasil, como reservatório para o predador Euseius citrifolius Denmark \& Muma (Acari: Phytoseiidae). Rev. Bras. Zool. 21(3):453-458.

DAUD, R.D. \& FERES, R.J.F. 2005. Diversidade e flutuação populacional de ácaros (Acari) em Mabea fistulifera Mart. (Euphorbiaceae) de dois fragmentos de mata estacional semidecídua em São José do Rio Preto. Neotrop. Entomol. 34(2):191-201.

DEMITE, P.R. \& FERES, R.J.F. 2005. Influência de vegetação vizinha na distribuição de ácaros em seringal (Hevea brasiliensis Muell. Arg., Euphorbiaceae) em São José do Rio Preto, São Paulo, Brasil. Neotrop. Entomol. 34(5):829-836.

DEMITE, P.R. \& FERES, R.J.F. 2007. Ocorrência e flutuação populacional de ácaros (Acari) associados a seringais (Hevea brasiliensis Muell. Arg.) vizinhos de fragmentos de Cerrado. Neotrop. Entomol. 36:117-127.

FERES, R.J.F. \& MORAES, G.J. De. 1998. Phytoseiid mites (Acari: Phytoseiidae) from wood areas in the State of São Paulo, Brazil. Sys. Appl. Acarol. 3:125-132.

FERES, R.J.F., ROSSA-FERES, D. De C., DAUD, R.D. \& SANTOS, R.S. 2002. Diversidade de ácaros (Acari, Arachnida) em seringueiras (Hevea brasiliensis Muell. Arg., Euphorbiaceae) na região noroeste do estado de São Paulo, Brasil. Rev. Bras. Zool. 19(1):137-144.
FERES, R.J.F., LOFEGO, A.C. \& OLIVEIRA, A.R. 2005. Ácaros plantícolas (Acari) da "Estação Ecológica do Noroeste Paulista", Estado de São Paulo, Brasil. Biota Neotrop. 5 (1): http://www.biotaneotropica.org.br/v5n1/pt/ abstract?article+BN00405012005. ISSN 1676-0603

FLECHTMANN, C.H.W. 1973. Lorryia formosa Cooremann, 1958 Um ácaro dos citros pouco conhecido no Brasil. Ciênc. Cult. 25(12):1179-1181

FLECHTMANN, C.H.W. 1975. Elementos de acarologia. Livraria Nobel S.A., São Paulo.

FLECHTMANN, C.H.W. 1981. Ácaros de importância agrícola. Livraria Nobel S.A., São Paulo.

GERSON, U. 1968. Five tydeid mites from Israel (Acarina: Prostigmata). Israel J. Zool. 17:191-198.

GERSON, U., SMILEY, R.L. \& OCHOA, R. 2003. Mites (Acari) for pest control. Blackwell Science Ltd., Oxford.

GRAY, J.S. 1987. Species-abundance patterns. In Organization of communities: Past and present. (J.H.R. Gee \& P.S. Giller, eds.). Blackwell Scientific Publications, Oxford, p.53-67.

HAMMER, Ø., HARPER, D.A.T. \& RYAN, P. D. 2001. PAST: Paleontological Statistics Software Package for Education and Data Analysis. Palaeontol. Electr. 4:9p.

HERNANDES, F.A. \& FERES, R.J.F. 2005. Two new species of Zetzellia Oudemans (Acari: Stigmaeidae) that threaten the concept of genera: disgeneric marriage? Zootaxa 1048:27-44.

HERNANDES, F.A. \& FERES, R.J.F. 2006. Biological cycle of Lorryia formosa Cooreman, 1958 (Acari, Tydeidae) on rubber trees leaves: a case of thelytoky. Exp. Appl. Acarol. 38(4):237-242.

HUSTON, M.A. 1995. Biological Diversity: The coexistence of species on changing landscapes. Cambridge University Press, Cambridge.

JEPPSON, L.R., KEIFER, H.H. \& BAKER, E.W. 1975. Mites injurious to economic plants. University of California Press, Berkeley.

KENNETT, C.E., FLAHERTY, D.L. \& HOFFMANN, R.W. 1979. Effect of wind-borne pollens on the population dynamics of Amblyseius hibisci (Acarina: Phytoseiidae). Entomophaga 24(1):83-98.

KRANTZ, G.W. 1978. A manual of acarology. Oregon State University Book Stores, Corvallis.

KREBS, C.J. 1999. Ecological Methodology. Ed. Adson Wesley Longman Inc., Menlo Park.

KRONKA, F.J.N, MATSUKUMA, C.K., NALON, M.A., DELCALI, I.H., ROSSI, M., MATTOS, I.F.A., SHIN-IKE, M.S. \& PONTINHAS, A.A.S. 1993. Inventário florestal do Estado de São Paulo. Instituto Florestal, São Paulo.

LINDQUIST, E.E. 1986. The world genera of Tarsonemidae (Acari: Heterostigmata): a morphological, phylogenetic and systematic revision, with a reclassification of family-group taxa in the Heterostigmata. Mem. Entomol. Soc. Canada 136:1-517.

LINDQUIST, E.E., SABELIS, M.W. \& BRUIN, J. 1996. Eriophyiod mites: Their biology, natural enemies and control. Elsevier Science B.V., Amsterdam.

LOFEGO, A.C. \& MORAES, G.J. De. 2005. Taxa de oviposição dos predadores Amblyseius acalyphus e Amblyseius neochiapensis (Acari: Phytoseiidae) com diferentes tipos de alimento. Arq. Inst. Biol. 72(3):379-382.

LOFEGO, A.C. \& MORAES, G.J. De. 2006. Ácaros (Acari) associados a mirtáceas (Myrtaceae) em áreas de Cerrado no estado de São Paulo com análise faunística das famílias Phytoseiidae e Tarsonemidae. Neotrop. Entomol. 35(6):731-746.

MAGURRAN, A. 1988. Ecological diversity and its measurement. Croom Helm Limited, London.

MCMURTRY J.A. \& CROFT, B.A. 1997. Life-styles of phytoseiid mites and their roles in biological control. Annu. Rev. Entomol. 42:291-321.

MORAES, G.J. De \& LIMA, H.C. 1983. Biology of Euseius concordis (Chant) (Acarina: Phytoseiidae) a predator of the tomato russet mite. Acarologia 24(3):251-255. 
ODUM, E.P. 1988. Ecologia. Editora Guanabara, Rio de Janeiro.

ROMERO, G.Q. \& BENSON, W.W. 2004. Leaf domatia mediate mutualism between mites and a tropical tree. Oecologia 140(4):609-616.

ROMERO, G.Q. \& BENSON, W.W. 2005. Biotic interactions of mites, plants and leaf domatia. Current Opinion in Plant Biology 8(4):436-440.

SMIRNOFF, W.A. 1957. An undescribed species of Lorryia fomosa (Acarina: Tydeidae) causing injury to citrus trees in Marrocco. J. Econ. Entomol. 50(3):361-362.

STRANGHETTI, V. \& RANGA, N.T. 1998. Levantamento florístico das espécies vasculares da floresta estacional mesófila semidecídua da Estação Ecológica de Paulo de Faria - SP. Rev. Bras. Bot. 21(3):289-298.
TRAVÉ, J., ANDRÉ, H.M., TABERLY, G. \& BERNINI, F. 1996. Les Acariens Oribates. AGAR/SIALF, Wavre.

WALTER, E.D. \& PROCTOR, H.C. 1998. Predatory mites in tropical Australia: Local Species Richness and complementarity. Biotropica 30(1):72-81

ZACARIAS, M.S. \& MORAES, G.J. De. 2002. Mite diversity (Arthropoda: Acari) on euphorbiaceous plants in three localities in the state of São Paulo. Biota Neotrop. 2(2): http://www.biotaneotropica.org.br/v2n2/pt/ abstract?article+BN00802022002

ZAR, J.H. 1999. Biostatistical analysis. $4^{\mathrm{a}}$ ed. Prentice-Hall, Inc., New Jersey. 